\title{
SIMULAÇÃO E CONTROLE GLOBAL DE UMA PLANTA TÍPICA PARA PRODUÇÃO DE BIODIESEL
}

\author{
B. F. da SILVA ${ }^{1}$, F. V. da SILVA ${ }^{1}$ e J. E. SCHMITZ ${ }^{1}$ \\ Universidade Estadual de Campinas, Faculdade de Engenharia Química \\ E-mail para contato: bfsfirmino@yahoo.com.br
}

\begin{abstract}
RESUMO - A produção de biodiesel tem crescido em importância nos últimos anos, sendo um importante substituto ao diesel. No Brasil, a maior parte da produção utiliza óleo de soja como matéria-prima, sendo a catálise homogênea alcalina a rota mais comum. O processo simulado representa um processo típico, no qual, óleo de soja é representado como trioleína pura na reação com metanol, utilizando soda cáustica como catalisador. Um modelo cinético rigoroso foi usado para representar a dinâmica do processo de transesterificação. Assim sendo, este trabalho tem como objetivo avaliar um sistema de controle global (plantwide) para um processo de produção de biodiesel. Foi utilizada a abordagem de simulação computacional para realizar a modelagem do processo, sendo escolhido o software Aspen Plus Dynamics para avaliar o comportamento dos controladores e das variáveis de interesse ao longo do tempo.
\end{abstract}

\section{INTRODUÇÃO}

O biodiesel é uma alternativa ao diesel proveniente do petróleo. Sua utilização crescente, busca a redução da emissão de poluentes e diminuição de dependência do petróleo. $\mathrm{Na}$ última década, normas regulatórias colocaram a obrigatoriedade da adição de $5 \%$ de biodiesel ao diesel vendido ao mercado brasileiro, o qual denomina-se diesel B5.

No final de 2012, a venda de diesel B5 chegou a 55,88 milhões de $\mathrm{m}^{3}$, representando $43 \%$ do consumo de combustíveis. Esta cifra representa um aumento de7\% em relação a 2011. Totalizou-se 2,73 milhões de $\mathrm{m}^{3}$ de biodiesel misturados no diesel B5, mostrando a grande demanda do biocombustível para o país (ANP, 2013, 2013b).

A mistura diesel e biodiesel, além de diminuir a dependência do combustível fóssil, traz vantagens ambientais, já que tem emissão de diversos poluentes reduzida com o aumento da porcentagem do biocombustível adicionada a mistura (EPA, 2002).

O termo biodiesel descreve uma mistura de substâncias semelhantes ao diesel que pode ser total ou parcialmente inserida como substituinte do diesel de origem fóssil. Comumente é composto por ésteres metílicos ou etílicos de ácidos graxos. Para sua obtenção, realiza-se a esterificação de ácidos graxos ou a transesterificação de triglicerídeos presentes em óleos vegetais e gordura animal.

A transesterificação ocorre através da reação do óleo vegetal com um álcool e geralmente na presença de um catalisador, o qual pode ser alcalino ou ácido em meio homogêneo ou heterogêneo, havendo ainda a possibilidade de realizar a reação através de 
catalisadores enzimáticos ou rotas não catalíticas envolvendo condições mais severas de temperatura e pressão.

\section{DESCRIÇÃO DO PROCESSO}

O processo proposto, simula a reação do óleo de soja com metanol, na presença de $\mathrm{NaOH}$ como catalisador. A escolha da rota foi embasada no mercado brasileiro, que tem cerca de $70 \%$ da produção através do óleo de soja, segundo a ANP (2013) e apresenta o metanol como álcool mais utilizado devido ao custo e qualidade final do biodiesel.

Para fins de simulação, devido à dificuldade de encontrar dados termodinâmicos para os componentes da mistura de triglicerídeos do óleo de soja, optou-se por realizar uma aproximação. Utilizou-se trioleína, um triglicerídeo composto por três ácidos oleicos. O peso molecular desse componente se aproxima da média presente na mistura de triglicerídeos do óleo de soja, sendo uma aproximação utilizada em diversos estudos. (Wang, 2008; Kiss,2011; Zhang et al., 2012).

Para o modelo cinético da reação utilizou-se o trabalho de Noureddini e Zhu (1997). Os autores propuseram um conjunto de reações reversíveis, como pode-se observar na Figura 1. Os parâmetros cinéticos da transesterificação do óleo de soja com metanol e catalisador homogêneo básico, foram ajustados para o modelo de Arrhenius conforme a Tabela 1.

$$
\begin{gathered}
\mathrm{TG}+\mathrm{CH}_{3} \mathrm{OH} \underset{k_{2}}{\stackrel{k_{1}}{\rightleftarrows}} \mathrm{DG}+\mathrm{R}_{1} \mathrm{COOCH}_{3} \\
\mathrm{DG}+\mathrm{CH}_{3} \mathrm{OH} \underset{k_{4}}{\stackrel{k_{3}}{\rightleftarrows}} \mathrm{MG}+\mathrm{R}_{2} \mathrm{COOCH}_{3} \\
\mathrm{MG}+\mathrm{CH}_{3} \mathrm{OH} \underset{k_{6}}{\stackrel{k_{5}}{\rightleftarrows}} \mathrm{GL}+\mathrm{R}_{2} \mathrm{COOCH}_{3}
\end{gathered}
$$

Figura 1- Reação de transesterificação, na qual TG, DG, MG e GL são triglicerídeos, diglicerídios, monoglicerídeos e glicerol.

Tabela 1- Parâmetros cinéticos da reação de transesterificação do óleo de soja.

\begin{tabular}{|l|c|c|}
\hline & \multicolumn{2}{|c|}{ Óleo de Soja1(Noureddini e Zhu,1997) } \\
\hline & $\begin{array}{c}\text { Energia de } \\
\text { Ativação (cal/mol) }\end{array}$ & $\begin{array}{c}\text { Constante da taxa } \\
\left(\mathrm{L} / \mathrm{mol} \text { min) a } 50^{\circ} \mathrm{C}\right.\end{array}$ \\
\hline TG --> DG & $\mathrm{E} 1=13145$ & $\mathrm{k} 1=0,05$ \\
\hline DG --> TG & $\mathrm{E} 2=9932$ & $\mathrm{k} 2=0,11$ \\
\hline DG --> MG & $\mathrm{E} 3=19860$ & $\mathrm{k} 3=0,215$ \\
\hline MG --> DG & $\mathrm{E} 4=14639$ & $\mathrm{k} 4=1,228$ \\
\hline MG --> GL & $\mathrm{E} 5=6421$ & $\mathrm{k} 5=0,242$ \\
\hline GL --> MG & E6 $=9588$ & $\mathrm{k} 6=0,007$ \\
\hline
\end{tabular}

O processo engloba a reação em três reatores CSTR sequenciais com alimentação de metanol em cada reator, o qual encontra-se em excesso em proporção de 9:1 necessária para 
obter a pureza final dos produtos, conforme pode ser observado na Figura 2. O catalisador é incorporado em cada reator em solução alcóolica. Após as saídas dos dois primeiros reatores ocorre uma separação inicial dos produtos em dois decantadores (D100 e D101), os quais separam uma fase rica em glicerol para realizar a purificação deste subproduto por destilação e a fase rica com biodiesel segue para o próximo reator.

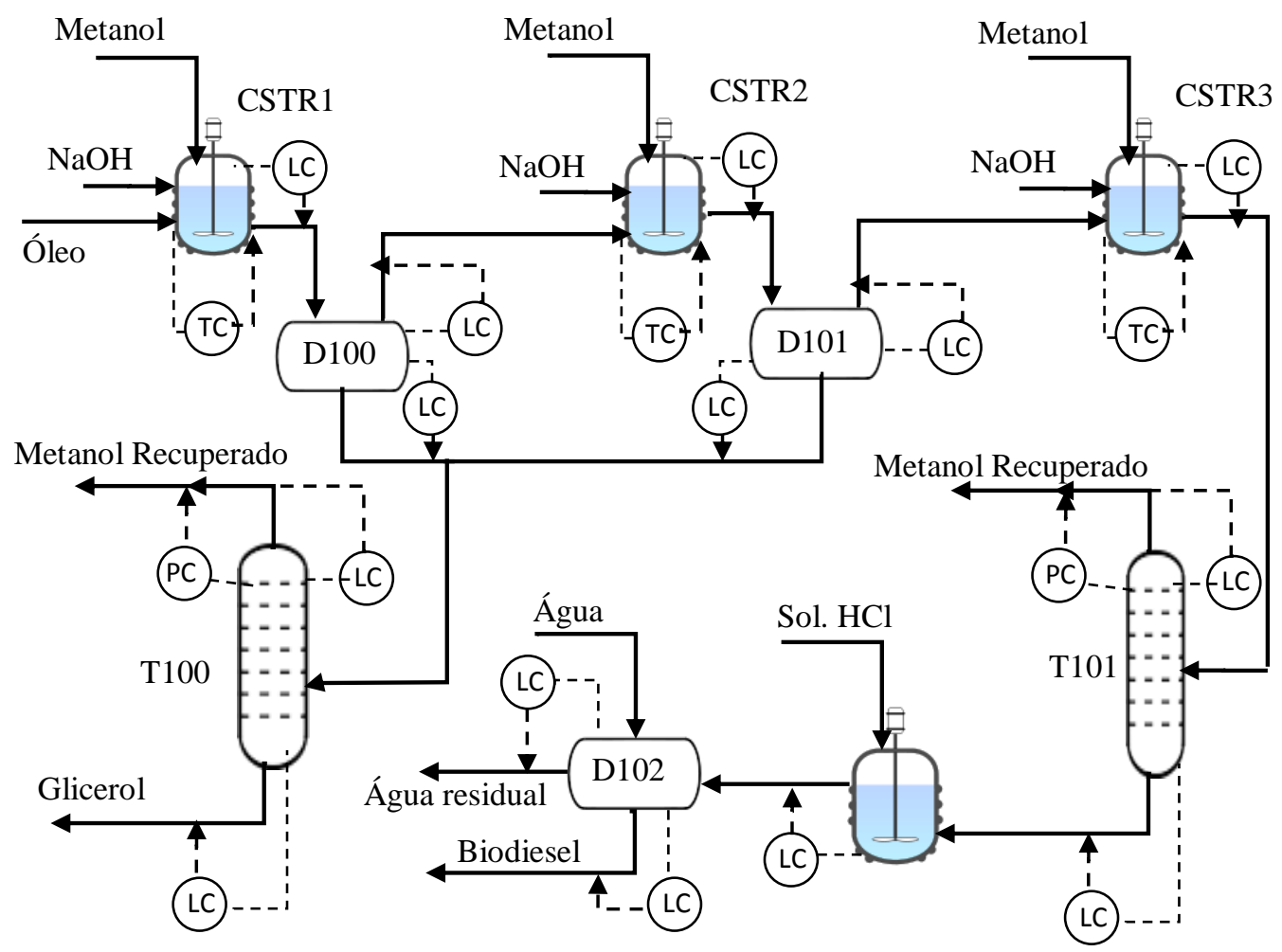

Figura 2 - Fluxograma do processo

As colunas de destilação são implementadas de modo a obter uma pureza necessária para um padrão internacional de comercialização, estabelecendo como restrição $96,5 \%$ de pureza para o biodiesel, valor este fixado pelo padrão europeu EN 14214, o qual influenciou o padrão brasileiro.

Para a coluna de destilação T100, responsável pela recuperação do metanol e do glicerol, foi utilizado 10 pratos com alimentação no $4^{\circ}$ prato. A pressão de operação o condensador foi de $20 \mathrm{kPa}$. A coluna necessitou trabalhar com vácuo para atingir a separação desejada sem ultrapassar o limite de temperatura de $150{ }^{\circ} \mathrm{C}$, na qual começa a ocorrer a degradação do glicerol.

Já a recuperação do biodiesel, ocorreu na coluna de destilação T101, na qual utilizou-se 5 pratos, com alimentação no $3^{\circ}$ prato. A pressão de operação do condensador ficou em 50 $\mathrm{kPa}$ para garantir que a coluna não ultrapassasse $250{ }^{\circ} \mathrm{C}$, temperatura na qual pode ocorrer a degradação dos ésteres. 
Após a destilação, a corrente rica em ésteres passar por um neutralizador, que reage uma solução de $\mathrm{HCl}$ com o $\mathrm{NaOH}$ residual, gerando água e $\mathrm{NaCl}$. Estes resíduos são eliminados na etapa seguinte de lavagem, na qual água é adicionada e ocorre a separação em um separador bifásico.

\section{METODOLOGIA}

Definiu-se a capacidade da planta em aproximadamente 200.000 toneladas por ano, produzindo biodiesel dentro dos padrões de comercialização presentes na norma europeia. Para atingir essa meta de produção, estabeleceu-se o valor de $28 \mathrm{kmol} / \mathrm{h}$ de trioleína como corrente de entrada.

O processo inicialmente foi simulado no software comercial Aspen Plus, através do qual obteve-se o regime permanente do processo. Para avaliar o comportamento ao longo do tempo, a simulação foi exportada para o software Aspen Plus Dynamics, o qual possibilitou a análise do regime transiente.

Para o cálculo das propriedades termodinâmicas das substâncias do processo foi utilizado o pacote termodinâmico UNIQUAC, com a determinação dos parâmetros faltantes pelo método de contribuição de grupos UNIFAC. Esta consideração foi realizada em outros estudos, tais como os trabalhos de Zhang et al. (2012) e Kiss (2011).

Foram realizadas perturbações na entrada de trioleína do processo de $-3 \%,-5 \%,-8 \%$, $3 \%, 5 \%$ e $8 \%$ para o fluxo molar. O tempo de simulação foi estabelecido até um novo regime permanente. Controladores foram implementados focando-se na planta como um todo, priorizando-se os objetivos de controle, restrições e controles complementares para alguns equipamentos.

\section{RESULTADOS E DISCUSSÕES}

Os controladores implementados são mostrados na Figura 2 na descrição do processo e os valores dos seus parâmetros de controle estão listados na Tabela 2. A sintonia foi realizada através do método de Zigler-Nichols implementado no software Aspen Plus Dynamics. Escolheu-se utilizar somente controladores PID para esse processo, tendo como hipótese que não há limitação para seu uso, ou seja, supõe-se que esteja disponível poder computacional suficiente para sua utilização. Soma-se a isto a ampla presença destes controladores na indústria, sendo o controle PID o mais comum (Desborough e Miller, 2002).

Os controles aplicados ao processo mostraram-se adequados, tendo em vista que conseguiram manter os set-points das principais variáveis. A coluna T101, a qual é responsável pela recuperação do metanol e purificação do biodiesel, foi considerado um ponto crítico do processo, já que impacta diretamente na qualidade do produto. Atingiu-se a pureza de $98,48 \%$ de ésteres no biodiesel para alimentação inicial de trioleína, resultado acima do previsto pela norma EN 14214 que regula o biocombustível. 
Tabela 2- Parâmetros de sintonia dos controladores.

\begin{tabular}{|c|c|c|c|}
\hline Controlador & Ganho (\%/\%) & $\begin{array}{c}\text { Constante de } \\
\text { tempo integral } \\
\text { (min) }\end{array}$ & $\begin{array}{c}\text { Constante de } \\
\text { tempo derivativo } \\
\text { (min) }\end{array}$ \\
\hline Pressão do condensador T100 & 0,51 & 2,40 & 0,60 \\
\hline Nível do condensador T100 & 0,24 & 4,36 & 1,09 \\
\hline Nível do refervedor T100 & 0,21 & 7,10 & 1,78 \\
\hline Pressão do condensador T101 & 0,19 & 2,40 & 0,60 \\
\hline Nível do condensador T101 & 0,06 & 2,40 & 0,60 \\
\hline Nível do refervedor T101 & 0,06 & 2,40 & 0,60 \\
\hline Nível da fase 1 do D100 & 90,95 & 3,50 & 0,87 \\
\hline Nível da fase 2 do D100 & 84,80 & 2,40 & 0,60 \\
\hline Nível da fase 1 do D101 & 0,52 & 2,56 & 5,00 \\
\hline Nível da fase 2 do D101 & 0,89 & 3,60 & 0,90 \\
\hline Nível do CSTR 1 & 81,91 & 2,47 & 0,62 \\
\hline Temperatura do CSTR 1 & 7,51 & 2,45 & 0,61 \\
\hline Nível do CSTR 2 & 89,07 & 2,46 & 0,62 \\
\hline Temperatura do CSTR 2 & 5,62 & 2,46 & 0,62 \\
\hline Nível do CSTR 3 & 64,42 & 3,68 & 0,92 \\
\hline Temperatura do CSTR 3 & 5,21 & 2,45 & 0,60 \\
\hline & & & \\
\hline & & & \\
\hline
\end{tabular}

Para as perturbações de 3\%,5\% e $8 \%$ no fluxo molar na entrada de trioleína, notou-se que a pureza do biodiesel sofre leve queda seguida de uma subida até a estabilização em um patamar superior ao inicial, porém, dentro dos limites desejados para a variável, este resultado pode ser visto na Figura 3. A natureza sequencial da reação aliado a alteração na razão entre metanol e óleo promove este comportamento na curva de pureza.

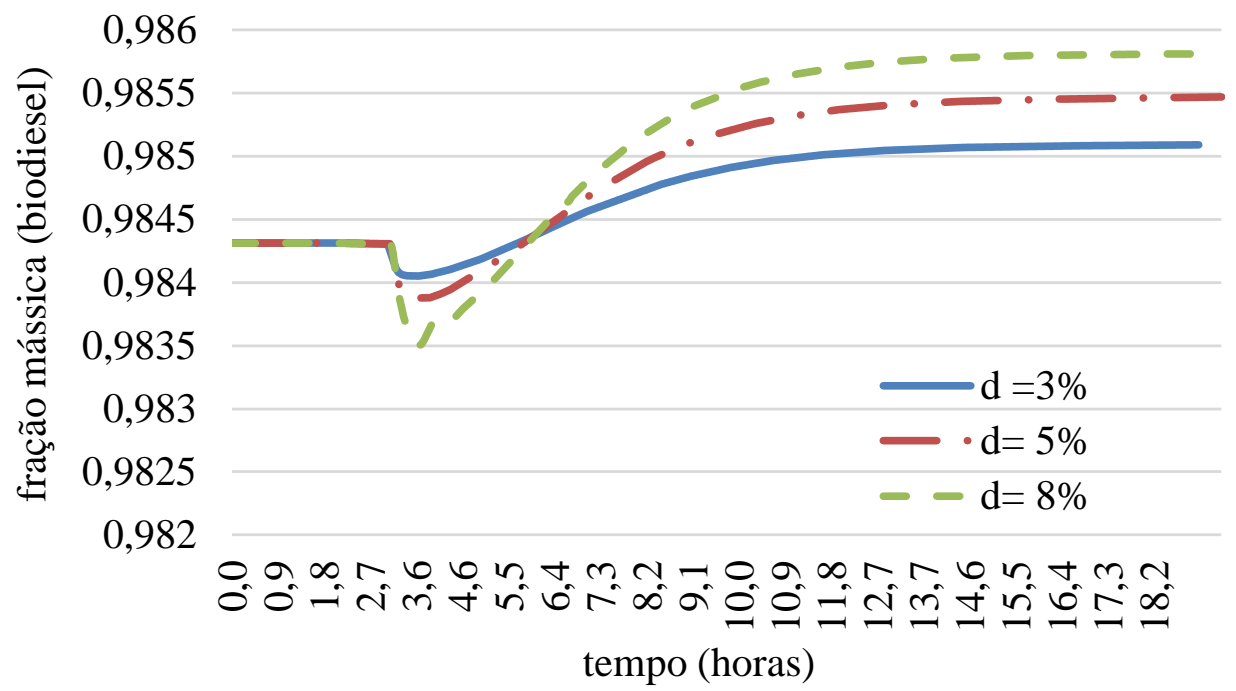

Figura 3 - Comportamento da pureza do biodiesel devido às perturbações de acréscimos do fluxo molar na corrente de trioleína. 
Para as perturbações em que há queda do fornecimento de trioleína em -3\%, -5\% e $8 \%$ no fluxo molar, observa-se comportamento contrário, estabilizando-se em um novo regime permanente com uma pureza menor que a inicial. Este efeito acompanha o decréscimo na corrente de óleo, conforme se verifica na Figura 4.

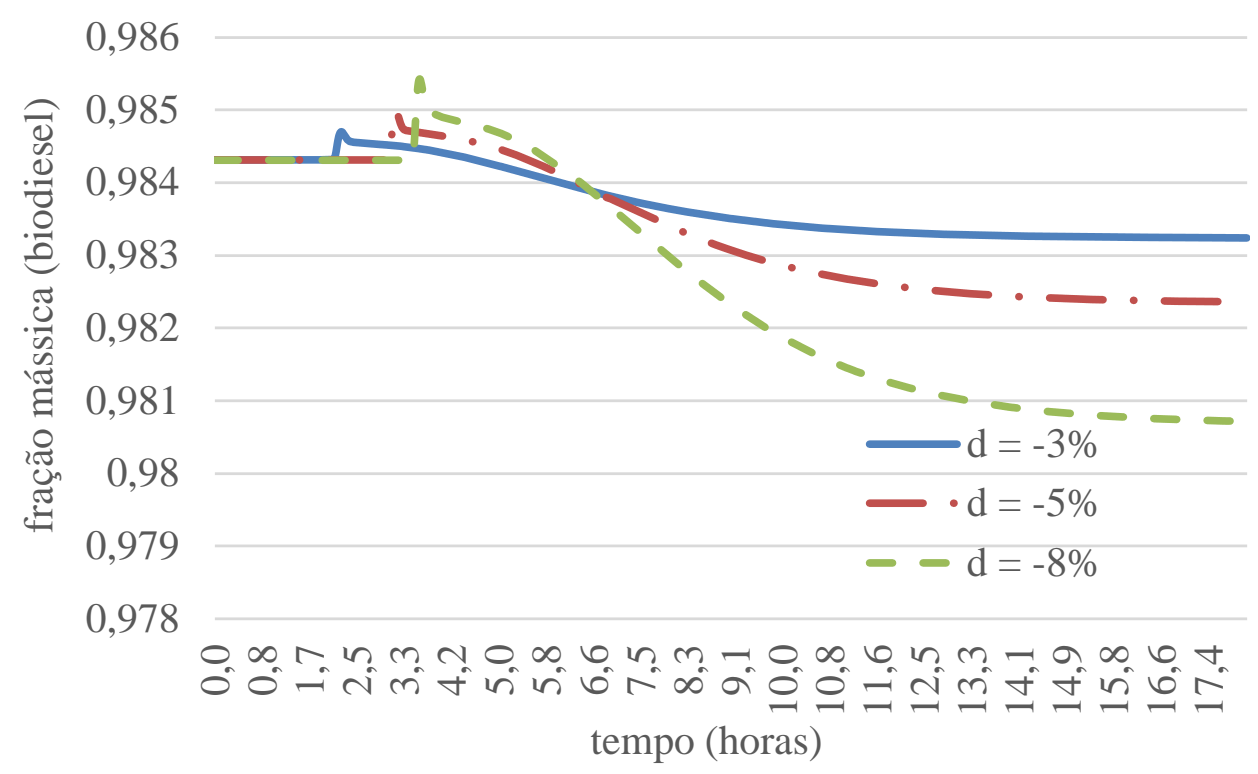

Figura 4 - Comportamento da pureza do biodiesel devido às perturbações de decréscimo do fluxo molar na corrente de trioleína.

A coluna de purificação de biodiesel T101 é um ponto crítico do processo, já que afeta diretamente a qualidade do produto final e tem a necessidade do controle da pressão do condensador, o qual trabalha com vácuo para evitar uma temperatura muito alta na separação e a degradação dos ésteres do biodiesel.

O controle da pressão foi realizado através da taxa de calor no condensador, ou seja, do fluxo de utilidade fria do mesmo. O condensador foi simulado de modo a obter uma saída subresfriada a $41,5^{\circ} \mathrm{C}$.

O comportamento das variáveis controladas e manipuladas da coluna de purificação de biodiesel pode ser visto na Figura 5, na qual aplicou-se um distúrbio no fluxo molar de trioleína de $-3 \%$. Nota-se uma rápida resposta e curto tempo de estabilização para a sintonia realizada. Para as demais perturbações estudadas obteve-se um resultado semelhante. 

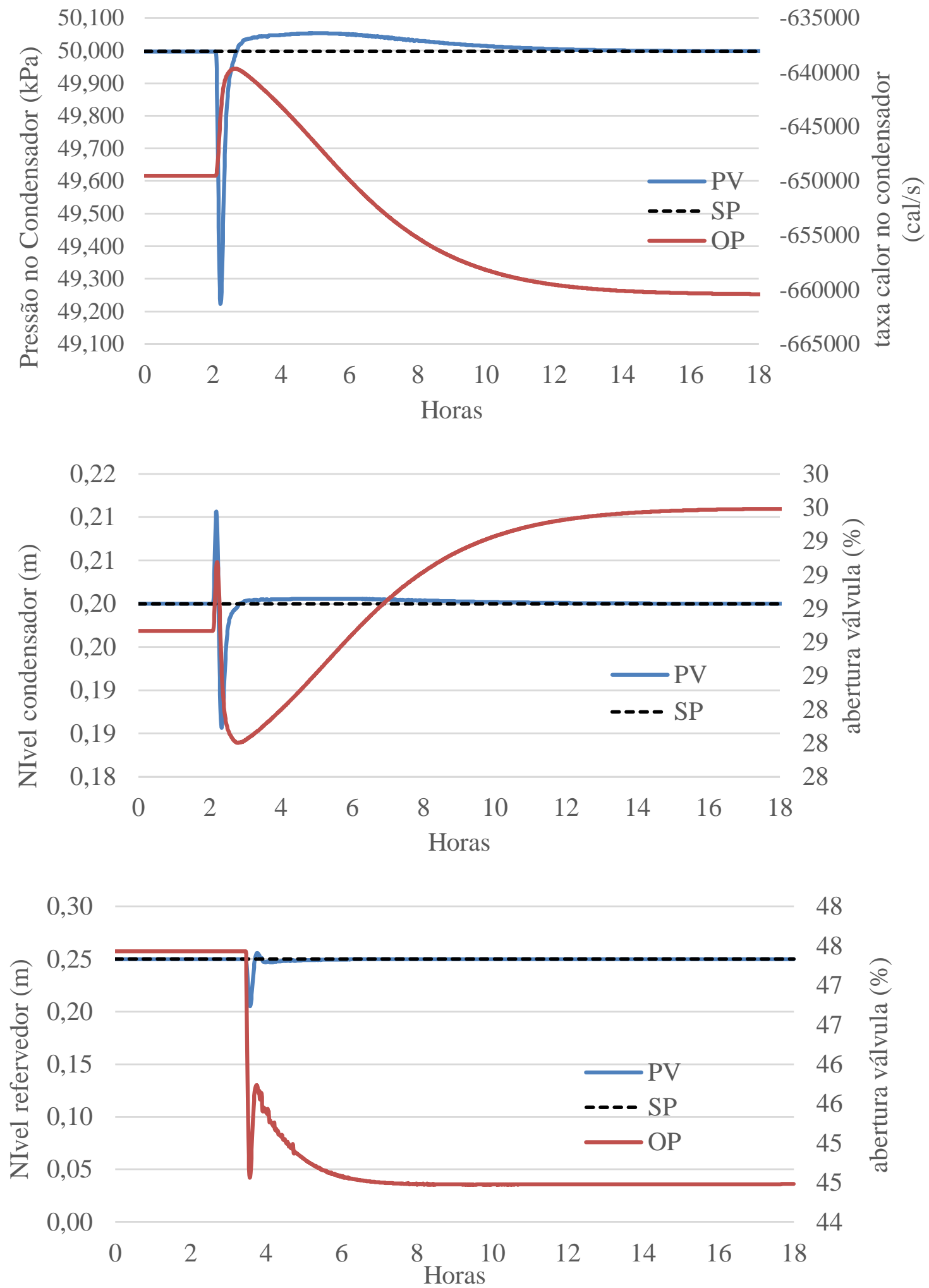

Figura 5 - Gráficos do comportamento dos controladores da coluna de purificação de biodiesel T101 (PV- Variável de processo; SP - set point; OP-Variável manipulada) 


\section{CONCLUSÕES}

O trabalho apresentou uma simulação da rota reacional mais abrangente da indústria. Utilizou-se cinética rigorosa, com parâmetros ajustados ao modelo cinético de Arrhenius, em um conjunto de reatores CSTR em série. A simulação atingiu as restrições estipuladas para a produção do biodiesel, tal como a pureza em padrão comercial. Um sistema de controle para a planta inteira foi estabelecido, no qual alguns controladores foram analisados, verificando-se que foi possível o controle do processo para as perturbações testadas. Como sequência do trabalho, faz-se necessário à implementação de outras propostas de controle através de metodologias orientadas para o controle de planta inteira (plantwide), assim como de indicadores globais para realizar comparação da performance das propostas de controle.

\section{AGRADECIMENTOS}

Os autores agradecem ao apoio da Coordenação de Aperfeiçoamento de Pessoal de Nível Superior - CAPES e a Faculdade de Engenharia Química, a qual apoia o desenvolvimento científico e a formação de pessoal qualificado.

\section{REFERÊNCIAS}

ANP. Boletim mensal de biodiesel: mês de dezembro de 2012. 2013. Disponível em:<http://www.anp.gov.br >. Acessado em: 25/02/2013.

ANP. Boletim mensal de combustíveis: mês de fevereiro de 2013. 2013b. Disponível em:<http://www.anp.gov.br >. Acessado em: 25/02/2013.

DESBOROUGH, L., MILLER, R. "Increasing customer value of industrial control performance monitoring - Honeywell's experience". InAIChE symposium series (pp. 169189). New York; American Institute of Chemical Engineers; 1998, 2002.

KISS A. A. "Heat-integrated reactive distillation process for synthesis of fatty esters" .Fuel Processing Technology, 92(7), 1288-1296, 2011.

NOUREDDINI, N., ZHU, D. "Kinetics of transesterification of soybean oil". Journal of American Oil Chemists' Society, 74, 1457-1463, 1997.

ZHANG, C.; RANGAIAH, G. P.; KARIWALA, V. Plantwide Control: Recent Developments and Applications. Cap. 14: Design and Plantwide Control of a Biodiesel Plant, p. 293, 2012.

WANG, T.Process simulation, integration and optimization of blending of petrodiesel with biodiesel.2008. Tese de Doutorado.Texas. A\&M University. 